\title{
ON THE LIE ALGEBRA OF A BURNSIDE GROUP OF EXPONENT 5
}

\author{
EUGENE F. KRAUSE AND KENNETH W. WESTON
}

Abstract. A. Kostrikin proved that $R(5,2)$ has order at most $5^{34}$ and is nilpotent of class at most 13 . He verified this by showing that the freest Lie ring $L$ on 2 generators of characteristic 5 which satisfies the 4th Engel condition must be nilpotent of class at most 13. He then appealed to the correspondence between Lie rings and groups, given by the associated Lie ring of a group, to complete his proof. We studied Kostrikin's calculations and considered a collection of matrices based on them. From these, we were able to construct $L$ with the use of a Univac 1107 computer and verify that $L$ is nilpotent of class exactly 13 with order exactly $5^{34}$. Hence, modulo the conjecture made by Kostrikin, Sanov and others that $L$ is the associated Lie ring of $R(5,2)$, the order of $R(5,2)$ is exactly 5 s.

1. Introduction. If $B(5,2)$ is the freest group of exponent 5 on 2 generators then its associated Lie ring, $\mathscr{L}(B)$, is a 2 generator Lie algebra over $\mathrm{GF}(5)$ satisfying the 4 th Engel condition [1, p. 329]. In fact, Kostrikin, Sanov, Zassenhaus, and others have conjectured that $\mathscr{L}(B)$ is the freest such Lie algebra.

Let $L(5,2,4)$ be the freest Lie algebra over $G F(5)$ which is generated by 2 elements and satisfies the 4 th Engel condition. Kostrikin [3] proved that $L(5,2,4)$ has dimension either 33 or 34 and hence has order $5^{33}$ or $5^{34}$. If the conjecture about $\mathcal{L}(B)$ is correct then it follows that the order of $B(5,2)$ is $5^{33}$ or $5^{34}$.

In this paper we show, with the aid of a Univac 1107 computer, that the dimension of $L(5,2,4)$ is, in fact, 34 and hence, modulo the conjecture, the order of $B(5,2)$ is precisely $5^{34}$. We actually do more; we realize $L(5,2,4)$ as a 34 dimensional column space over $G F(5)$ and present its multiplication table (Table 3). Although we make extensive use of Kostrikin's calculations, the result is verified to be correct on the computer.

2. Matrix representation. If $L$ is a Lie algebra over a field $F$, $\mathcal{E}(L)$ is its (associative) algebra of linear operators, and $\mathcal{L}(\mathcal{E}(L)$ ) is the Lie algebra of $\mathcal{E}(L)$, then the adjoint mapping, ad, from $L$ to

Received by the editors January 29, 1970 and, in revised form, May 19, 1970.

AMS 1969 subject classifications. Primary 2040, 2015, 2008; Secondary 1730, 0280.

Key words and phrases. Restricted Burnside group of exponent 5, associated Lie ring, freest Lie algebra, generators, GF(5), 4th Engel condition. 
$\mathcal{L}(\mathcal{E}(L)$ ) is an antihomomorphism [2, p. 18] ("anti" because we have chosen to write operators on the left rather than the right). If $\beta$ $=\left\{m_{1}, m_{2}, \cdots, m_{n}\right\}$ is a basis for $L$ then the usual coordinate and matrix mappings, $\kappa_{\beta}$ and $\mu_{\beta}$, are isomorphisms from $L$ to $M_{n \times 1}$ and $\mathcal{E}(L)$ to $M_{n \times n}$ respectively, where $M_{p \times q}$ denotes the set of all $p$ by $q F$-matrices and the multiplication, $\mathrm{X}$, in $M_{n \times 1}$ is defined by

$$
c_{1} \times c_{2}=\mu_{\beta} \text { ad } \kappa_{\beta}^{-1}\left(c_{2}\right) \cdot c_{1} \text {. }
$$

In particular if $\left\{\lambda_{1}, \lambda_{2}, \cdots, \lambda_{n}\right\}$ is the natural basis for $M_{n \times 1}$ then

$$
\lambda_{i} \times \lambda_{j}=i \text { th column of } \mu_{\beta} \operatorname{ad}\left(m_{j}\right) .
$$

The composition, $\mu_{\beta}$ ad, from $L$ to $\mathscr{L}\left(M_{n \times n}\right)$, the Lie algebra of the matrix algebra, is an antihomomorphism and hence

$$
\mu_{\beta} \operatorname{ad}\left(l_{1} \circ l_{2} \circ \cdots \circ l_{k}\right)=(-1)^{k+1}\left[\mu_{\beta} \operatorname{ad}\left(l_{1}\right), \mu_{\beta} \operatorname{ad}\left(l_{2}\right), \cdots, \mu_{\beta} \operatorname{ad}\left(l_{k}\right)\right] .
$$

In particular if $L$ is generated by $x, y, \cdots$ and if we have the matrices $\mu_{\beta} \operatorname{ad}(x), \mu_{\beta} \operatorname{ad}(y), \cdots$ then (3) provides a simple formula for computing $\mu_{\beta} \operatorname{ad}(m)$ where $m=m(x, y, \cdots)$ is any monomial in $L$.

3. Exploiting Kostrikin's calculations. Kostrikin [2] considered the freest Lie algebra $L$ over GF(5) generated by 2 elements, $x$ and $y$, satisfying the 4th Engel condition. He was able to find a spanning set of 34 monomials $m_{i}=m_{i}(x, y)$ in $x$ and $y$ (Table 1 ) and was able to prove the independence of the first 33 . He was not able to prove that all 34 constitute a basis.

TABLE 1. Kostrikin's spanning set for $L$

\begin{tabular}{lll}
\hline \hline$m_{1}=y$ & $m_{12}=y x^{2} y x^{2}$ & $m_{23}=x y^{2} x y x y^{3}$ \\
$m_{2}=x$ & $m_{13}=x y^{2} x y x$ & $m_{24}=y x^{2} y x y x^{3}$ \\
$m_{3}=x y$ & $m_{14}=y x^{3} y^{2}$ & $m_{26}=x y^{3} x^{3} y^{2}$ \\
$m_{4}=x y^{2}$ & $m_{15}=x y^{2} x y x y$ & $m_{26}=y x^{3} y^{3} x^{2}$ \\
$m_{5}=y x^{2}$ & $m_{16}=y x^{2} y x y x$ & $m_{27}=y x^{2} y x^{2} y^{3}$ \\
$m_{6}=x y^{3}$ & $m_{17}=y x^{3} y^{3}$ & $m_{28}=x y^{2} x y^{2} x^{3}$ \\
$m_{7}=y x^{3}$ & $m_{18}=x y^{3} x^{3}$ & $m_{29}=x y^{3} x^{3} y^{3}$ \\
${ }^{*} m_{8}=x y^{2} x$ & $m_{19}=x y^{2} x y x y^{2}$ & $m_{30}=y x^{3} y^{3} x^{3}$ \\
$m_{9}=x y^{2} x y$ & $m_{20}=y x^{2} y x y x^{2}$ & $m_{31}=x y^{3} x^{3} y^{2} x$ \\
$m_{10}=y x^{2} y x$ & $m_{21}=x y^{3} x^{3} y$ & $m_{32}=x y^{3} x^{3} y^{3} x$ \\
$m_{11}=x y^{2} x y^{2}$ & $m_{22}=y x^{2} y x^{2} y^{2}$ & $m_{33}=y x^{3} y^{3} x^{3} y$ \\
& & $m_{34}=y x^{3} y^{3} x^{3} y^{2}$ \\
\hline
\end{tabular}

$\left({ }^{*} x y^{2} x\right.$ means $\left.((x \circ y) \circ y) \circ x.\right)$ 
TABLE 2. The matrices $M_{1}=\left(y_{i j}\right), M_{2}=\left(x_{i j}\right)$.

All entries are 0 except

\begin{tabular}{lllll}
\hline$y_{3,2}=1$ & $y_{21,16}=3$ & $x_{8,1}=4$ & $x_{21,17}=1$ \\
$y_{4,3}=1$ & $y_{21,18}=1$ & $x_{5,3}=4$ & $x_{22,15}=1$ \\
$y_{6,4}=1$ & $y_{22,16}=4$ & $x_{7,5}=1$ & $x_{24,20}=1$ \\
$y_{8,5}=4$ & $y_{23,19}=1$ & $x_{8,4}=1$ & $x_{26,21}=1$ \\
$y_{9,8}=1$ & $y_{25,21}=1$ & $x_{9,6}=3$ & $x_{26,22}=4$ \\
$y_{10,7}=3$ & $y_{27,22}=1$ & $x_{10,8}=4$ & $x_{27,19}=1$ \\
$y_{11,9}=1$ & $y_{28,20}=1$ & $x_{12,10}=1$ & $x_{28,22}=4$ \\
$y_{14,10}=2$ & $y_{29,25}=1$ & $x_{13,9}=1$ & $x_{29,23}=4$ \\
$y_{15,13}=1$ & $y_{30,24}=4$ & $x_{15,11}=4$ & $x_{30,26}=1$ \\
$y_{16,12}=4$ & $y_{31,26}=1$ & $x_{16,14}=3$ & $x_{31,25}=1$ \\
$y_{17,14}=1$ & $y_{31,28}=4$ & $x_{17,11}=3$ & $x_{31,27}=4$ \\
$y_{18,12}=3$ & $y_{32,31}=2$ & $x_{18,13}=2$ & $x_{32,29}=1$ \\
$y_{19,15}=1$ & $y_{33,30}=1$ & $x_{20,16}=1$ & $x_{33,31}=2$ \\
& $y_{34,33}=1$ & $x_{21,15}=4$ & $x_{34,32}=1$ \\
\hline
\end{tabular}

We heuristically assumed $\beta=\left\{m_{1}, m_{2}, \cdots, m_{34}\right\}$ to be a basis and, using many of Kostrikin's calculations, proceeded to find the two matrices

$$
M_{1}=\mu_{\beta} \operatorname{ad}\left(m_{1}\right), \quad M_{2}=\mu_{\beta} \operatorname{ad}\left(m_{2}\right)
$$

(Table 2). Then equation (3) and the Univac were employed to determine the additional matrices

$$
M_{i}=\mu_{\beta} \operatorname{ad}\left(m_{i}\right), \quad i=3,4, \cdots, 34 .
$$

After using equation (2) to fill in the multiplication table for the (natural) basis elements of $M_{34 \times 1}$ (Table 3) we observed, from Table 3 , that $\lambda_{1}, \lambda_{2}$ generate $M_{34 \times 1}$. Finally we turned again to the computer to verify that $M_{34 \times 1}$ is indeed a Lie algebra satisfying the 4th Engel condition. (A program for verifying the Jacobi and Engel conditions is on file in the Computing Science Library of the University of Notre Dame.)

Thus we arrived at a 2 generator Lie algebra over GF(5) which satisfies the 4th Engel condition and has dimension 34-namely, $M_{34 \times 1}$. Our assumption that $\beta=\left\{m_{1}, m_{2}, \cdots, m_{34}\right\}$ be a basis showed us how to construct it. Its properties, however, were all verified independent of that assumption.

Now since $L=L(5,2,4)$ it follows that the dimension of $L$ must be at least 34 . But the spanning set $\beta$ consists of 34 elements. Thus the dimension of $L$ is $34, \beta$ is a basis, and $L \cong M_{34 \times 1}$ under $\kappa_{\beta}$. 
TABLE 3. Lie multiplication table for $M_{34 \times 1}$. Missing entries are to be determined by skew-symmetry

\begin{tabular}{|c|c|c|c|c|c|c|c|c|}
\hline & $\lambda_{1}$ & $\lambda_{2}$ & $\lambda_{3}$ & $\lambda_{t}$ & $\lambda_{s}$ & $\lambda_{0}$ & $\lambda_{T}$ & $\lambda_{8}$ \\
\hline$\lambda_{1}$ & 0 & $4 \lambda_{8}$ & $4 \lambda_{4}$ & $4 \lambda_{6}$ & $\lambda_{8}$ & 0 & $2 \lambda_{10}$ & $4 \lambda$, \\
\hline$\lambda_{2}$ & & 0 & $\lambda_{\delta}$ & $4 \lambda_{8}$ & $4 \lambda_{7}$ & $2 \lambda_{0}$ & 0 & $\lambda_{10}$ \\
\hline$\lambda_{2}$ & & & 0 & $2 \lambda_{0}$ & $3 \lambda_{10}$ & $2 \lambda_{11}$ & $3 \lambda_{12}$ & $\lambda_{18}+2 \lambda_{14}$ \\
\hline$\lambda_{8}$ & & & & 0 & $\lambda_{18}+3 \lambda_{14}$ & 0 & $\lambda_{16}+3 \lambda_{18}$ & $3 \lambda_{16}+4 \lambda_{17}$ \\
\hline$\lambda_{s}$ & & & & & 0 & $\lambda_{16}+3 \lambda_{12}$ & 0 & $2 \lambda_{10}+\lambda_{18}$ \\
\hline$\lambda_{6}$ & & & & & & 0 & $4 \lambda_{21}+3 \lambda_{22}$ & $\lambda_{10}$ \\
\hline$\lambda_{z}$ & & & & & & & 0 & $4 \lambda_{20}$ \\
\hline$\lambda_{8}$ & & & & & & & & 0 \\
\hline \multicolumn{9}{|l|}{$\lambda_{0}$} \\
\hline \multicolumn{9}{|l|}{$\lambda_{10}$} \\
\hline \multicolumn{9}{|l|}{$\lambda_{11}$} \\
\hline \multicolumn{9}{|l|}{$\lambda_{12}$} \\
\hline \multicolumn{9}{|l|}{$\lambda_{12}$} \\
\hline \multicolumn{9}{|l|}{$\lambda_{14}$} \\
\hline \multicolumn{9}{|l|}{$\lambda_{\text {w }}$} \\
\hline \multicolumn{9}{|l|}{$\lambda_{16}$} \\
\hline \multicolumn{9}{|l|}{$\lambda_{17}$} \\
\hline \multicolumn{9}{|l|}{$\lambda_{18}$} \\
\hline \multicolumn{9}{|l|}{$\lambda_{10}$} \\
\hline \multicolumn{9}{|l|}{$\lambda_{20}$} \\
\hline \multicolumn{9}{|l|}{$\lambda_{21}$} \\
\hline \multicolumn{9}{|l|}{$\lambda_{22}$} \\
\hline \multicolumn{9}{|l|}{$\lambda_{23}$} \\
\hline \multicolumn{9}{|l|}{$\lambda_{2 t}$} \\
\hline \multicolumn{9}{|l|}{$\lambda_{2 s}$} \\
\hline \multicolumn{9}{|l|}{$\lambda_{26}$} \\
\hline \multicolumn{9}{|l|}{$\lambda_{z}$} \\
\hline \multicolumn{9}{|l|}{$\lambda_{28}$} \\
\hline \multicolumn{9}{|l|}{$\lambda_{20}$} \\
\hline \multicolumn{9}{|l|}{$\lambda_{30}$} \\
\hline \multicolumn{9}{|l|}{$\lambda_{21}$} \\
\hline \multicolumn{9}{|l|}{$\lambda_{32}$} \\
\hline \multicolumn{9}{|l|}{$\lambda_{n}$} \\
\hline$\lambda_{n}$ & & & & & & & & \\
\hline
\end{tabular}


Table 3-(Continued)

\begin{tabular}{|c|c|c|c|c|c|c|}
\hline$\lambda_{0}$ & $\lambda_{10}$ & $\lambda_{11}$ & $\lambda_{12}$ & $\lambda_{18}$ & $\lambda_{14}$ & $\lambda_{\mathbf{u}}$ \\
\hline $4 \lambda_{11}$ & $3 \lambda_{14}$ & 0 & $\lambda_{16}+2 \lambda_{18}$ & $4 \lambda_{16}$ & $4 \lambda_{17}$ & $4 \lambda_{10}$ \\
\hline $4 \lambda_{12}$ & $4 \lambda_{12}$ & $\lambda_{13}+2 \lambda_{17}$ & 0 & $3 \lambda_{18}$ & $2 \lambda_{16}$ & $\lambda_{21}+4 \lambda_{2}$ \\
\hline $3 \lambda_{16}+3 \lambda_{17}$ & $2 \lambda_{16}+2 \lambda_{18}$ & $\lambda_{10}$ & $4 \lambda_{20}$ & $2 \lambda_{21}+\lambda_{23}$ & $2 \lambda_{21}+3 \lambda_{23}$ & $\lambda_{23}$ \\
\hline $2 \lambda_{10}$ & $4 \lambda_{21}+2 \lambda_{22}$ & $\lambda_{23}$ & $\lambda_{26}+4 \lambda_{28}$ & $\lambda_{2 s}+\lambda_{2 z}$ & $3 \lambda_{25}+3 \lambda_{n}$ & $2 \lambda_{20}$ \\
\hline $2 \lambda_{21}+3 \lambda_{23}$ & $2 \lambda_{20}$ & $\lambda_{25}+4 \lambda_{27}$ & $\lambda_{24}$ & $\lambda_{28}+\lambda_{28}$ & $3 \lambda_{28}+3 \lambda_{28}$ & $3 \lambda_{n}$ \\
\hline$\lambda_{23}$ & $3 \lambda_{2 s}+\lambda_{2 z}$ & 0 & $2 \lambda_{31}$ & $4 \lambda_{22}$ & $4 \lambda_{20}$ & 0 \\
\hline $3 \lambda_{28}+\lambda_{22}$ & $\lambda_{24}$ & $2 \lambda_{31}$ & 0 & $3 \lambda_{20}$ & $2 \lambda_{30}$ & $4 \lambda_{\mathfrak{n}}$ \\
\hline $4 \lambda_{25}+\lambda_{27}$ & $\lambda_{26}+4 \lambda_{28}$ & $4 \lambda_{20}$ & $\lambda_{80}$ & $3 \lambda_{21}$ & $\lambda_{81}$ & $2 \lambda_{32}$ \\
\hline \multirow[t]{7}{*}{0} & 0 & 0 & $3 \lambda_{\mathfrak{z}}$ & $4 \lambda_{82}$ & $4 \lambda_{z z}$ & 0 \\
\hline & 0 & $3 \lambda_{z z}$ & 0 & $3 \lambda_{\mathfrak{w}}$ & $2 \lambda_{z}$ & $3 \lambda_{\mu}$ \\
\hline & & 0 & 0 & 0 & 0 & 0 \\
\hline & & & 0 & 0 & $\mathbf{0}$ & 0 \\
\hline & & & & 0 & 0 & 0 \\
\hline & & & & & 0 & $\mathbf{0}$ \\
\hline & & & & & & 0 \\
\hline & & & & & & \\
\hline & & & & & & \\
\hline & & & & & & \\
\hline & & & & & & \\
\hline & & & & & & \\
\hline & & & & & & \\
\hline & & & & & & \\
\hline & & & & & & \\
\hline & & & & & & \\
\hline & & & & & & \\
\hline & & & & & & \\
\hline & & & & & & \\
\hline & & & & & & \\
\hline & & & & & & \\
\hline & & & & & & \\
\hline & & & & & & \\
\hline & & & & & & \\
\hline & & & & & & \\
\hline
\end{tabular}


Table 3-(Continued)

\begin{tabular}{|c|c|c|c|c|c|c|c|c|c|}
\hline & $\lambda_{16}$ & $\lambda_{17}$ & $\lambda_{18}$ & $\lambda_{19}$ & $\lambda_{20}$ & $\lambda_{21}$ & $\lambda_{22}$ & $\lambda_{28}$ & $\lambda_{24}$ \\
\hline$\lambda_{1}$ & $2 \lambda_{21}+\lambda_{22}$ & 0 & $4 \lambda_{21}$ & $4 \lambda_{23}$ & $4 \lambda_{28}$ & $4 \lambda_{25}$ & $4 \lambda_{27}$ & 0 & $\lambda_{80}$ \\
\hline$\lambda_{2}$ & $4 \lambda_{20}$ & $4 \lambda_{21}$ & 0 & $4 \lambda_{27}$ & $4 \lambda_{24}$ & $4 \lambda_{26}$ & $\lambda_{28}+\lambda_{28}$ & $\lambda_{29}$ & 0 \\
\hline$\lambda_{3}$ & $4 \lambda_{26}$ & $4 \lambda_{26}$ & $\lambda_{26}$ & $4 \lambda_{29}$ & $\lambda_{30}$ & 0 & $4 \lambda_{31}$ & 0 & 0 \\
\hline$\lambda_{4}$ & $3 \lambda_{31}$ & $4 \lambda_{20}$ & $\lambda_{31}$ & 0 & $3 \lambda_{33}$ & $\lambda_{82}$ & $\lambda_{82}$ & 0 & 0 \\
\hline$\lambda^{5}$ & $2 \lambda_{30}$ & $\lambda_{\mathbf{3 1}}$ & $4 \lambda_{30}$ & $3 \lambda_{32}$ & 0 & $\lambda_{33}$ & $3 \lambda_{33}$ & 0 & 0 \\
\hline$\lambda_{6}$ & $4 \lambda_{32}$ & 0 & $\lambda_{32}$ & 0 & 0 & 0 & 0 & 0 & 0 \\
\hline$\lambda_{7}$ & 0 & $\lambda_{33}$ & 0 & 0 & 0 & 0 & 0 & 0 & 0 \\
\hline$\lambda_{8}$ & $3 \lambda_{33}$ & $3 \lambda_{32}$ & $2 \lambda_{33}$ & 0 & 0 & 0 & 0 & 0 & 0 \\
\hline$\lambda_{9}$ & $3 \lambda_{34}$ & 0 & $2 \lambda_{34}$ & 0 & 0 & 0 & 0 & 0 & 0 \\
\hline$\lambda_{10}$ & 0 & $2 \lambda_{34}$ & 0 & 0 & 0 & 0 & 0 & 0 & 0 \\
\hline$\lambda_{11}$ & 0 & 0 & 0 & 0 & 0 & 0 & 0 & 0 & 0 \\
\hline$\lambda_{12}$ & 0 & 0 & 0 & $\mathbf{0}$ & 0 & 0 & 0 & 0 & 0 \\
\hline$\lambda_{18}$ & 0 & 0 & 0 & 0 & 0 & 0 & 0 & 0 & 0 \\
\hline$\lambda_{14}$ & 0 & 0 & 0 & 0 & 0 & 0 & 0 & $\mathbf{0}$ & 0 \\
\hline$\lambda_{15}$ & 0 & 0 & 0 & 0 & 0 & 0 & 0 & o & 0 \\
\hline$\lambda_{16}$ & 0 & 0 & 0 & 0 & 0 & 0 & 0 & 0 & 0 \\
\hline$\lambda_{17}$ & & 0 & 0 & 0 & 0 & 0 & 0 & 0 & 0 \\
\hline$\lambda_{18}$ & & & 0 & 0 & 0 & 0 & 0 & 0 & 0 \\
\hline$\lambda_{19}$ & & & & 0 & 0 & 0 & 0 & 0 & 0 \\
\hline$\lambda_{20}$ & & & & & 0 & 0 & 0 & 0 & 0 \\
\hline$\lambda_{21}$ & & & & & & 0 & 0 & 0 & 0 \\
\hline$\lambda_{22}$ & & & & & & & 0 & 0 & 0 \\
\hline$\lambda_{23}$ & & & & & & & & 0 & 0 \\
\hline$\lambda_{24}$ & & & & & & & & & 0 \\
\hline \multicolumn{10}{|l|}{$\lambda_{26}$} \\
\hline \multicolumn{10}{|l|}{$\lambda_{28}$} \\
\hline \multicolumn{10}{|l|}{$\lambda_{27}$} \\
\hline \multicolumn{10}{|l|}{$\lambda_{28}$} \\
\hline \multicolumn{10}{|l|}{$\lambda_{29}$} \\
\hline \multicolumn{10}{|l|}{$\lambda_{30}$} \\
\hline \multicolumn{10}{|l|}{$\lambda_{31}$} \\
\hline \multicolumn{10}{|l|}{$\lambda_{32}$} \\
\hline \multicolumn{10}{|l|}{$\lambda_{38}$} \\
\hline$\lambda_{34}$ & & & & & & & & & \\
\hline
\end{tabular}


Table 3-(Concluded)

\begin{tabular}{|c|c|c|c|c|c|c|c|c|c|}
\hline$\lambda_{26}$ & $\lambda_{26}$ & $\lambda_{n}$ & $\lambda_{28}$ & $\lambda_{28}$ & $\lambda_{30}$ & $\lambda_{31}$ & $\lambda_{32}$ & $\lambda_{8 z}$ & $\lambda_{34}$ \\
\hline $4 \lambda_{29}$ & $4 \lambda_{81}$ & 0 & $\lambda_{31}$ & 0 & $4 \lambda_{33}$ & $3 \lambda_{32}$ & 0 & $4 \lambda_{34}$ & 0 \\
\hline $4 \lambda_{31}$ & $4 \lambda_{30}$ & $\lambda_{31}$ & 0 & $4 \lambda_{32}$ & 0 & $3 \lambda_{33}$ & $4 \lambda_{34}$ & 0 & 0 \\
\hline $4 \lambda_{32}$ & $\lambda_{33}$ & $2 \lambda_{32}$ & $3 \lambda_{s 3}$ & 0 & 0 & 0 & 0 & 0 & 0 \\
\hline 0 & $\lambda_{\boldsymbol{M}}$ & 0 & $3 \lambda_{34}$ & 0 & 0 & 0 & 0 & 0 & 0 \\
\hline$\lambda_{\mu}$ & 0 & $3 \lambda_{\mu}$ & 0 & 0 & 0 & 0 & 0 & 0 & 0 \\
\hline 0 & 0 & 0 & 0 & 0 & 0 & 0 & 0 & 0 & 0 \\
\hline 0 & 0 & 0 & 0 & 0 & 0 & 0 & 0 & 0 & 0 \\
\hline 0 & 0 & 0 & 0 & 0 & 0 & 0 & 0 & 0 & 0 \\
\hline 0 & 0 & 0 & 0 & 0 & 0 & 0 & 0 & 0 & 0 \\
\hline 0 & 0 & 0 & 0 & 0 & 0 & 0 & 0 & 0 & 0 \\
\hline 0 & 0 & 0 & 0 & 0 & 0 & 0 & 0 & 0 & 0 \\
\hline 0 & 0 & 0 & 0 & 0 & 0 & 0 & 0 & 0 & 0 \\
\hline 0 & 0 & 0 & 0 & 0 & 0 & 0 & 0 & 0 & 0 \\
\hline 0 & 0 & 0 & 0 & 0 & 0 & 0 & 0 & 0 & 0 \\
\hline 0 & 0 & 0 & 0 & 0 & 0 & 0 & 0 & 0 & 0 \\
\hline 0 & 0 & 0 & 0 & 0 & 0 & 0 & 0 & 0 & 0 \\
\hline 0 & 0 & 0 & 0 & 0 & 0 & 0 & 0 & 0 & 0 \\
\hline 0 & 0 & 0 & 0 & 0 & 0 & 0 & 0 & 0 & 0 \\
\hline 0 & 0 & 0 & 0 & 0 & 0 & 0 & 0 & 0 & 0 \\
\hline 0 & 0 & 0 & 0 & 0 & 0 & 0 & 0 & 0 & 0 \\
\hline 0 & 0 & 0 & 0 & 0 & 0 & 0 & 0 & 0 & 0 \\
\hline 0 & 0 & 0 & 0 & 0 & 0 & 0 & 0 & 0 & 0 \\
\hline 0 & 0 & 0 & 0 & 0 & 0 & 0 & 0 & 0 & 0 \\
\hline 0 & 0 & 0 & 0 & 0 & 0 & 0 & 0 & 0 & 0 \\
\hline \multirow[t]{10}{*}{0} & 0 & 0 & 0 & 0 & 0 & 0 & 0 & 0 & 0 \\
\hline & 0 & 0 & 0 & 0 & o & 0 & 0 & 0 & 0 \\
\hline & & 0 & 0 & 0 & 0 & 0 & 0 & 0 & 0 \\
\hline & & & 0 & 0 & 0 & 0 & 0 & 0 & 0 \\
\hline & & & & 0 & 0 & 0 & 0 & 0 & 0 \\
\hline & & & & & 0 & 0 & 0 & 0 & 0 \\
\hline & & & & & & 0 & 0 & 0 & 0 \\
\hline & & & & & & & 0 & 0 & 0 \\
\hline & & & & & & & & 0 & 0 \\
\hline & & & & & & & & & 0 \\
\hline
\end{tabular}




\section{REFERENCES}

1. M. Hall, Jr., The theory of groups, Macmillan, New York, 1959. MR 21 \#1996.

2. N. Jacobson, Lie algebras, Interscience Tracts in Pure and Appl. Math., no. 10, Interscience, New York, 1962. MR $26 \# 1345$.

3. A. I. Kostrikin, The restricted Burnside problem for exponent 5, Izv. Akad. Nauk SSSR Ser. Mat. 19 (1955), 233-244. (Russianj MR 17, 126; 1436.

University of Michigan, Ann Arbor, Michigan 48104

Marquette University, Milwaukee, Wisconsin 53233 